\title{
Birt-Hogg-Dubé syndrome and laparoscopic surgery: \\ Is this an anaesthetic cause for concern?
}

\author{
F.Lucci', M.Dauri', M.B.Silvi', J.W.Freeman', G. Tisone ${ }^{2}$ \\ IDepartment of Anaesthesia and Intensive care, University of Rome Tor Vergata \\ 2Department of Hepatobiliary and Liver Transplant, University of Rome Tor Vergata
}

\section{Background:}

Birt-Hogg-Dubé Syndrome (BHDS) is a rare autosomal dominant disorder characterized by hair follicle hamartomas, renal tumours and spontaneous pneumothoraces. We present a case of a patient with BHDS and his anaesthetic management.

\section{Case Report:}

A 65-year-old man presented with abdominal pain. Computerized tomography (CT-scan) revealed multiple cysts in both lungs and an abdominal lesion in the upper pole of his left kidney for which he required laparoscopic nephrectomy.

Family anamnesis revealed a relative with BirtHogg-Dubé Syndrome (BHDS) who had presented with a pneumothorax. No additional organ lesions were detected. Genetic testing confirmed the diagnosis of BHDS in both individuals.

In our patient general anaesthesia was performed cognisant of the risks of laparoscopic surgery in a patient with multiple lungs cysts and the risk of pneumothorax. Low Intermittent pressure ventilation with no PEEP was used following induction with Propofol, Sufentanil and Rocuronium. Intra abdominal pressure was kept to a minimum. Recovery was uneventful and he was transferred to the
Intensive Care Unit. Post-operative chest $X$ ray and arterial blood gases confirmed that the anaesthesia had been uneventful.

\section{Discussion:}

BHDS is extremely rare with a prevalence of I in 200,000 but is probably under diagnosed. The Implications of not diagnosing this condition or missing the presence of lung cysts and bullae preoperatively could have serious consequences under anaesthesia. These risks are accentuated by raised intra abdominal pressure with pneumoperitoneum and Trendelenburg position. The case highlights that anaesthetic management and surgical manipulation can be challenging and life threatening. Its imperative that a joint approach to management is undertaken.

\section{Learning points:}

Preoperative assessment of all patients is important. Rare conditions probably occur more frequently undiagnosed than expected. A multi professional approach should always be adopted in patient care.

\section{References:}

I. Birt AR, Hogg GR, Dubé WJ. Hereditary multiple fibrofolliculomas with trichodiscomas and acrochordons. Arch. Dermatol. 1977; I I3(I2): I674-7.

2. Menko, FH et al. Birt-Hogg-Dubé syndrome: diagnosis and management. Lancet Oncol 2009; 10, I $199-1206$

3. Ozer et al. Multiple lung cysts and Birt-Hogg-Dubé Syndrome: Management of anaesthesia and surgery.

Gulhane Med J. 2012;54:302-305

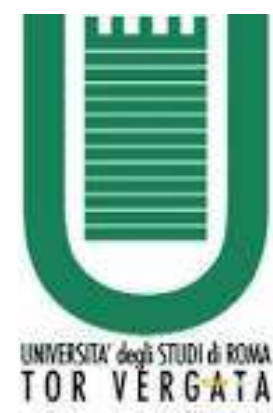

